\title{
Exercise induced hypoglycaemic hyperinsulinism
}

T Meissner, T Otonkoski, R Feneberg, B Beinbrech, S Apostolidou, I Sipilä, F Schaefer, E Mayatepek

\begin{abstract}
Background-Hyperinsulinism in childhood is often caused by genetic defects involving the regulation of insulin secretion leading to recurrent episodes of hypoglycaemia. We report two patients with exercise induced hypoglycaemia.

Methods-Standardised short exercise tests with frequent blood glucose and plasma insulin measurements were performed in the patients and young healthy controls.

Results-Short term exercise resulted in insulin induced hypoglycaemia 15 to 50 minutes after the end of exercise. A massive burst of insulin secretion was observed within a few minutes of the start of exercise in both patients. By contrast glucose and insulin concentrations remained unchanged in healthy controls.

Conclusions-Hyperinsulinaemic hypoglycaemia after moderate physical exercise represents a rarely described phenotype of hyperinsulinism with an as yet unknown defect in the regulation of insulin secretion. It should be suspected in individuals with recurrent exercise related syncope or disturbance of consciousness.

(Arch Dis Child 2001;84:254-257)
\end{abstract}

Division of Metabolic and Endocrine Diseases, University Children's Hospital, Im Neuenheimer Feld 150, D-69120

Heidelberg, Germany

T Meissner

B Beinbrech

S Apostolidou

E Mayatepek

Division of Pediatric Nephrology,

Department of

General Pediatrics,

University Children's

Hospital

R Feneberg

F Schaefer

Hospital for Children and Adolescents, Haartman Institute, University of Helsinki, Finland

T Otonkoski

I Sipilä

Correspondence to: Dr Mayatepek ertan_mayatepek@ med.uni-heidelberg.de

Accepted 20 September 2000
Keywords: exercise; hypoglycaemia; hyperinsulinism; congenital hyperinsulinism

Hyperinsulinism is a disease phenotype characterised by increased insulin secretion leading to hypoglycaemia (blood glucose concentration less than $2.6 \mathrm{mmol} / \mathrm{l}$ ), coma, and potential brain damage if untreated. Diffuse as well as focal histological abnormalities of the pancreas have been described. Aynsley-Green and colleagues ${ }^{1}$ defined a disease characterised by severe non-ketotic hypoglycaemia with inappropriately raised plasma insulin concentrations presenting during the neonatal period. This severe form, with irregular hyperinsulinism, is usually called persistent hyperinsulinaemic hypoglycaemia of infancy (PHHI; OMIM 601820), nesidioblastosis, or familial hyperinsulinism (HI; OMIM 256450). Hyperinsulinism may be caused by a range of biochemical disturbances and molecular defects, ${ }^{2}$ such as a defective $\mathrm{K}_{\mathrm{ATP}}$ channel caused by mutations in the sulphonylurea receptor gene or the Kir6.2 gene, ${ }^{3}$ or an increased activity of $\beta$ cell glucokinase or glutamate dehydrogenase. ${ }^{45}$

We now report two patients with exercise induced hyperinsulinaemic hypoglycaemia, who represent a rarely recognised clinical phenotype.

\section{Patients and methods}

PATIENT 1

A 16 year old boy of Caucasian origin presented with a two year history of recurrent syncope. These episodes usually occurred a few minutes after vigorous exercise. Family history was unremarkable. Blood glucose measurements during one of these syncopes revealed severe hypoglycaemia (blood glucose $<1.6 \mathrm{mmol} / \mathrm{l}$; control range $3.0-5.5 \mathrm{mmol} / \mathrm{l}$ ) which responded rapidly to intravenous glucose. The patient was subsequently admitted to our hospital for further diagnostic evaluation. Physical examination, including psychomotor development, was completely normal. Repeatedly normal concentrations of ammonia excluded the syndrome of hyperammonaemia and hyperinsulinaemia. ${ }^{5}$ In the fasting state, he developed symptomatic hypoglycaemia after 12 hours. At this time blood glucose was 2.2 $\mathrm{mmol} / 1$ and insulin $20 \mathrm{mU} / 1$. A second fasting test also resulted in hypoglycaemia and confirmed hyperinsulinism. Of note, ketone bodies and free fatty acids were very low during hypoglycaemia.

We suspected an insulinoma but ultrasonography and magnetic resonance imaging of the pancreas were unrevealing. ${ }^{111}$ In octreotide scintigraphy was normal. Diagnostic laparotomy with manual investigation of the pancreas and intraoperative ultrasonography did not show any abnormalities. Several pancreatic biopsies showed normal pancreatic histology. We did not perform pancreatic resection and the patient declined further diagnostic work up to rule out a focal lesion, including pancreatic venous sampling. He was treated with diazoxide $(3.5 \mathrm{mg} / \mathrm{kg} /$ day $)$ and his blood glucose is stable so long as he avoids exercise.

\section{PATIENT 2}

This girl presented at the age of 11 months, when she was admitted with convulsions. Family history was unremarkable. She had probably suffered from concussion on the previous day and had been eating poorly. Blood glucose was $0.9 \mathrm{mmol} / 1$ on admission and rose to normal with intravenous glucose. Subsequently, she had several hospital admissions for hypoglycaemic episodes and syncope. Spontaneous blood glucose values in the range $0.9-2.0 \mathrm{mmol} / 1$ were measured several times during early childhood. Blood insulin concentration was never measured at the time of her repeated hypoglycaemic episodes. However, there was no hint of any other inborn metabolic disease. Subsequently, extended fasting did not provoke hypoglycaemia. 
At the age of 5 years her liver biopsy was morphologically normal. Magnetic resonance imaging of the pancreas was normal. Prolonged exercise, particularly swimming, seemed to cause hypoglycaemia like symptoms, different from exhaustion and fatigue, although biochemical hypoglycaemia was never documented. At the age of 15.4 years we performed a bicycle exercise test with monitoring of plasma glucose and insulin (fig 2). The test was stopped after 10 minutes and blood glucose concentration measurements 20 minutes later revealed hyperinsulinaemic hypoglycaemia, caused by a notable increase in insulin secretion.

\section{METHODS}

Study protocol

Different standardised exercise tests were performed in both patients to evaluate the cause of the exercise induced hypoglycaemia.

Patient 1-Exercise associated insulin and glucose profiles were studied during 100 minutes, starting five minutes before a five minute dumbbell exercise. The exercise test was performed three times under different conditions: without diazoxide after a four hour fast; with diazoxide $(3.5 \mathrm{mg} / \mathrm{kg} /$ day $)$ after a four hour fast; and with diazoxide $(3.5 \mathrm{mg} / \mathrm{kg} /$ day $)$ 45 minutes after a carbohydrate rich meal. Time interval between the three exercise tests was at least one week. Control values for the glucose and insulin response to five minutes of dumbbell exercise were obtained in four healthy individuals (age 21-31 years). In addition, baseline blood insulin and glucose concentrations without exercise were measured every 15 minutes for 12 hours (without diazoxide) or 24 hours (with diazoxide), respectively. The diurnal insulin profiles were compared to those of 11 young healthy controls investigated by the same protocol. The results are published elsewhere. ${ }^{6}$

Patient 2-A bicycle exercise test with 10 minutes of intensive cycling was performed, with glucose and insulin concentration measured every five minutes for 60 minutes. Control values for the glucose and insulin response to 10 minutes of bicycle exercise were obtained in four healthy individuals (age 21-31 years).

\section{Analytical methods}

One drop of each blood sample was immediately used for blood glucose concentration measurements with a precision plus analyser (Medisense, UK). We used this technique as we had to evaluate the glucose concentrations immediately during the testing. Portable glycaemia dosimeters are useful because of their high reproducibility (SD $0.15 \mathrm{mmol} / \mathrm{l}$ ), but have a lack of accuracy compared to laboratory based methods primarily influenced by the haematocrit. However, haematocrit was always stable and normal in our patients. The rest of the blood sample was stored on ice and centrifuged after clotting. The serum was stored at $-80^{\circ} \mathrm{C}$ until analysis. Serum insulin concentrations were determined using the insulin ELISA (enzyme linked immunosorbent assay; Dako
Diagnostika, Hamburg, Germany), a highly sensitive two site immunospecific ELISA with two monoclonal murine antibodies. The detection limit of the assay is $0.5 \mathrm{mU} / 1$. There is no cross reactivity with human $\mathrm{C}$ peptide and virtually none with human proinsulin $(0.3 \%)$, 32-33 split proinsulin (0.3\%), and des-31-32 split proinsulin $(0.5 \%)$. Each sample was assayed in duplicate.

\section{Results}

The endocrine work up confirmed mild hyperinsulinism with reduced fasting tolerance in patient 1 . The analysis of insulin secretion and glucose concentration was performed during basal conditions and without any medical treatment over a 12 hour daytime period; this showed a high mean insulin secretion (37.3 $\mathrm{mU} / 1$ versus $17.1 \mathrm{mU} / 1$ in controls) but no episodes of hypoglycaemia. Insulin secretion was normalised after treatment with diazoxide (18.1 mU/1; fig 1), with low insulin values at rest during the night and stable glucose concentrations despite the night fast. Patient 1 performed three exercise tests under different conditions (table 1). Maximum insulin concentrations were measured 8-12 minutes after the beginning of exercise, which was stopped after five minutes (fig 2). The patient became hypoglycaemic 21-50 minutes after the end of exercise. Neither diazoxide nor pre-exercise food intake changed the patient's susceptibility for exercise induced hypoglycaemia (table 1).

In patient 2 a short bicycle exercise test elicited a similar burst of insulin secretion, resulting in hypoglycaemia 20 minutes after the end
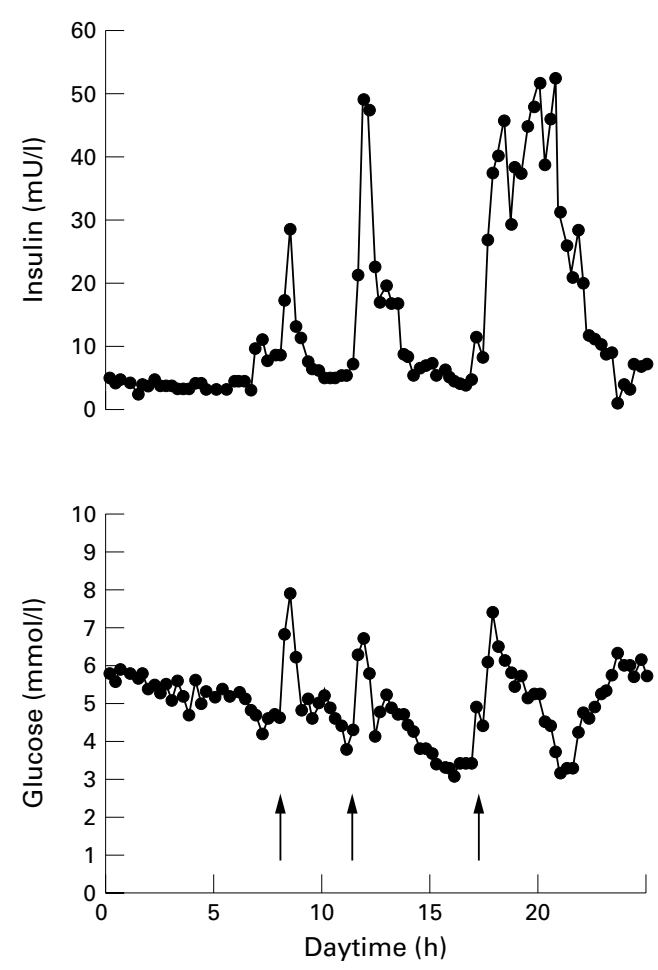

Figure 1 Twenty four hour insulin and glucose v time profile in patient 1 under basal conditions. With diazoxide treatment $(3.5 \mathrm{mg} / \mathrm{kg} / \mathrm{day})$ the glucose concentration was stable. Postprandial increases of glucose (arrows) are followed by a normal pattern of postprandial insulin release. 
Table 1 Insulin and glucose v time profile in patient $1(A, B, C)$ and healthy controls after five minutes of dumbbell exercise or 10 minutes of bicycle exercise

\begin{tabular}{llll}
\hline Exercise test & Study protocol & $\begin{array}{l}\text { Max. insulin } \\
(\mathrm{mU} / \mathrm{l})^{\star}\end{array}$ & $\begin{array}{c}\text { Min. glucose } \\
(\text { mmol/l })^{\star}\end{array}$ \\
\hline A & Without diazoxide, after 4 hours fast & $49(12 \mathrm{~min})$ & $1.9(30 \mathrm{~min})$ \\
B & With diazoxide, after 4 hours fast & $88(10 \mathrm{~min})$ & $1.7(54 \mathrm{~min})$ \\
C & With diazoxide, 45 min after carbohydrate rich meal & $209(8 \mathrm{~min})$ & $2.0(55 \mathrm{~min})$ \\
Dumbbell controls ( $=4)$ & After 4 hours fast & $3.5-13(1-44 \mathrm{~min})$ & $>3.5 \mathrm{mmol} / 1$ \\
Bicycle controls $(\mathrm{n}=4)$ & After 4 hours fast & $8.8-16(1-35 \mathrm{~min})$ & $>4.2 \mathrm{mmol} / 1$ \\
\hline
\end{tabular}

«Time after exercise in parentheses.

Patient 1
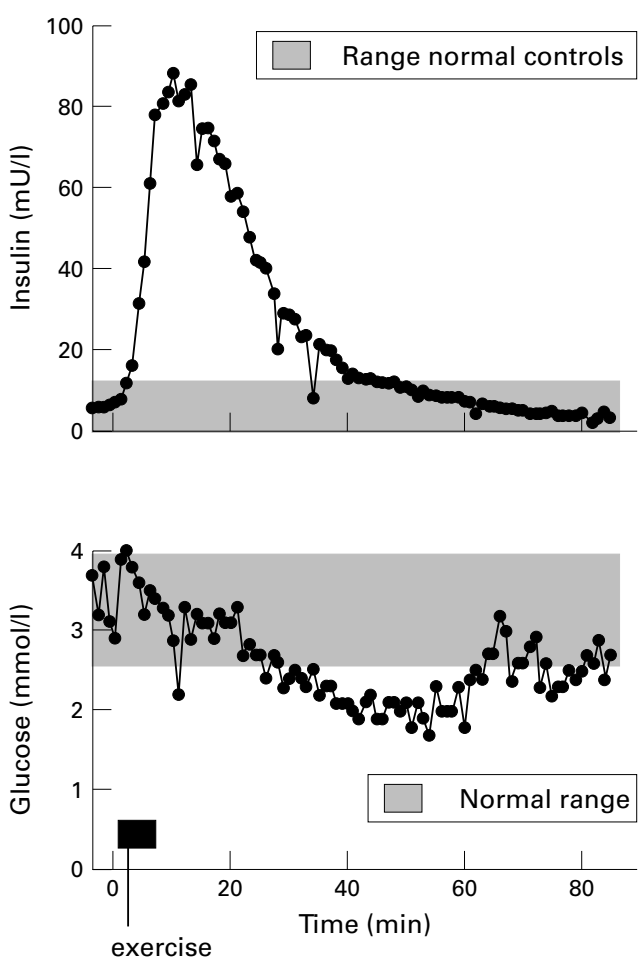

Patient 2
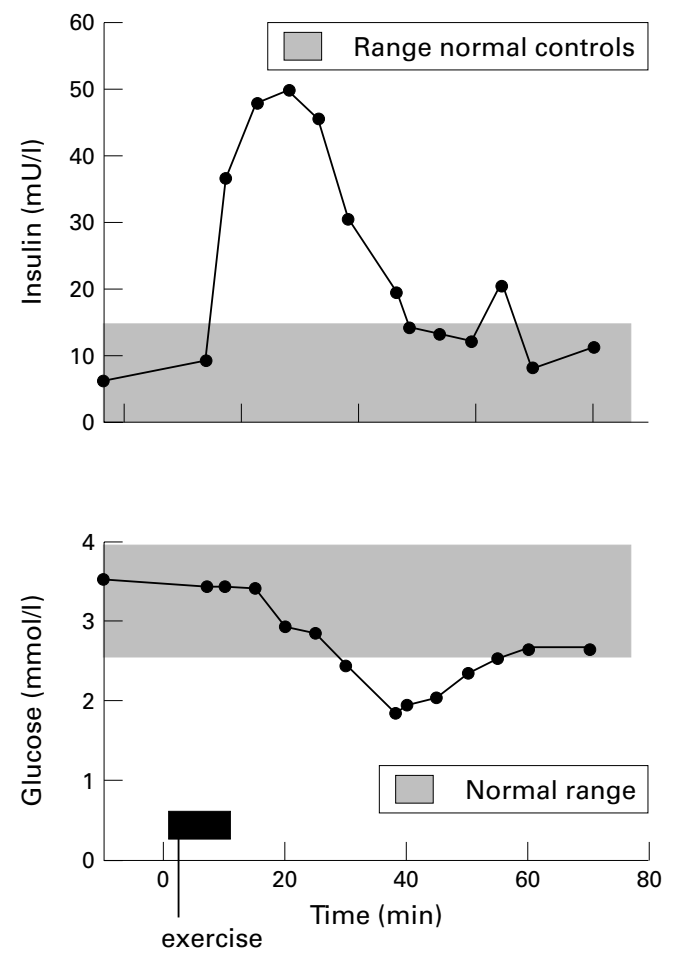

Figure 2 Exercise test. After five basal measurements at rest, patient 1 and four healthy young adult controls performed dumbbell exercise for five minutes. The insulin and glucose concentrations were measured every minute for 90 minutes. Patient 2 and four healthy controls underwent intensive bicycle exercise for 10 minutes. Insulin and glucose concentrations were measured every five minutes.

of exercise (fig 2). Healthy control subjects showed no major increase in blood insulin concentration after exercise (table 1, fig 2).

\section{Discussion}

These two children had recurrent exercise induced episodic syncope. Exercise tests confirmed hyperinsulinaemic hypoglycaemia in both. This phenotype differs from all previously described types of hyperinsulinism. It is characterised by mild exercise related hyperinsulinism with relatively good fasting tolerance of at least 12 hours without medical treatment, and highly increased insulin secretion after short term "anaerobic" exercise, resulting in symptomatic hypoglycaemia.

Remarkably, when we studied these children, only short, intensive exercise resulted in hypoglycaemia. We tested patient 1 three times to determine an adequate form of treatment. However, neither diazoxide nor food intake before exercise prevented hypoglycaemia. In this condition it is not clear to what extent insulin secretion is caused by exercise or food intake. Nevertheless, despite different treat- ment regimes, this patient still became hypoglycaemic after exercise.

Previous investigations had resulted in hyperinsulinaemic fasting hypoglycaemia in patient 1 , but it is important to note that he was not at rest during the fasting episode. He was walking, climbing stairs, etc, and this kind of physical exercise may have contributed to increased basal insulin secretion and fasting hypoglycaemia. After the night fast he always had stable blood glucose concentrations.

Frequent serial blood sampling revealed a burst of insulin secretion in response to exercise, resulting in a gradual lowering of blood glucose and overt hypoglycaemia 20-50 minutes after discontinuing the exercise. At that time, insulin concentrations were still increased in relation to low blood glucose concentrations.

Hyperinsulinism throughout the day was confirmed by frequent blood sampling. Treatment with diazoxide, in a low dose of 3.5 $\mathrm{mg} / \mathrm{kg} /$ day, lowered insulin concentrations to the normal range. Increasing diazoxide dosage resulted in hyperglycaemia and mild oedema. 
However, repeated exercise testing showed a persistent burst of insulin release during exercise, even during diazoxide medication.

Various biochemical disturbances and molecular defects have been described in hyperinsulinism. ${ }^{2}$ However, none of the hitherto identified pathogenetic mechanisms can explain exercise induced hyperinsulinism. Alterations in the $\mathrm{K}_{\mathrm{ATP}}$ channel activity of pancreatic $\beta$ cells caused by mutations in the SUR-1 or Kir6.2 genes result in a continuously aberrant release of insulin, which, in most cases is not regulated by metabolic signals. In addition, hyperinsulinaemia with focal pancreatic lesions has been related to a loss of maternal alleles in $11 \mathrm{p} 15$ and paternal SUR-1 mutations. ${ }^{78}$ Late onset hyperinsulinism after the neonatal period has been described in individuals with autosomal dominant inherited mutations in the glucokinase $^{4}$ or the glutamate dehydrogenase (GLUD-1) gene. ${ }^{5}$ So far, mutational analyses in our patients have been unremarkable. However, in all patients with known genetic defects resulting in hyperinsulinism the phenotype was clearly different from exercise induced hypoglycaemia. As our patients have been clinically stable without vigorous exercise latterly and there was no evidence of an insulinoma, pancreatic resection was not indicated.

A previous report described two siblings presenting with hyperinsulinism aggravated after vigorous exercise. ${ }^{9}$ These siblings had syncope after exercise at the age of 14 and 27 years, respectively. They might suffer from the same entity as our patients.

The underlying defect of this new phenotype entity is unknown but familial appearance in two siblings ${ }^{9}$ strongly suggests a genetic basis. Exercise induced hyperinsulinaemic hypoglycaemia should be included in the differential diagnosis of recurrent exercise related syncope and other disturbances of consciousness.

1 Aynsley-Green A, Polak JM, Bloom SR, et al. Nesidioblastosis of the pancreas: definition of the syndrome and the management of the severe neonatal hyperinsulinaemic hypoglycaemia. Arch Dis Child 1981;56:496-508.

2 Meissner T, Beinbrech B, Mayatepek E. Persistent hyperinsulinemic hypoglycaemia of infancy: molecular basis of heterogeneous disease. Hum Mutat 1999;13:351-61.

3 Thomas PM, Cote GJ, Wohllk N, et al. Mutations in the sulfonylurea receptor gene in familial persistent hyperinsulinemic hypoglycaemia of infancy. Science 1995;268: 426-9.

4 Glaser B, Kesavan P, Heyman M, et al. Familial hyperinsulinism caused by an activating glucokinase mutation. $N$ Engl F Med 1998;338:226-30.

5 Stanley CA, Lieu YK, Hsu BY, et al. Hyperinsulinism and hyperammonemia in infants with regulatory mutations of the glutamate dehydrogenase gene. $N$ Engl $f$ Med 1998;338:1352-7.

6 Feneberg R, Sparber M, Veldhuis JD, et al. Synchronous fluctuations of blood insulin and lactate concentrations in humans. F Clin Endocrinol Metab 1999;84:220-7.

7 Ryan F, Devaney D, Joyce C, et al. Hyperinsulinism: Ryan F, Devaney D, Joyce C, et al. Hyperinsulinism:
molecular etiology of focal disease. Arch Dis Child 1998;79: 445-7.

8 Verkarre V, Fournet JC, de Lonlay P, et al. Paternal mutation of the sulfonylurea receptor (SUR1) gene and maternal loss of $11 \mathrm{p} 15$ imprinted genes lead to persistent hyperinsulinism in focal adenomatous hyperplasia. 7 Clin Invest 1998;102:1286-91.

9 Burman WJ, McDermott MT, Bornemann M. Familial hyperinsulinism presenting in adults. Arch Intern Med 1992;152:2125-7. 